\title{
Relatively weakly open sets in closed balls of $C^{*}$-algebras
}

\author{
Julio Becerra Guerrero, Ginés López Pérez, and A. Rodríguez-Palacios
}

\begin{abstract}
Let $A$ be an infinite-dimensional $C^{*}$-algebra. We prove that every nonempty relatively weakly open subset of the closed unit ball $B_{A}$ of $A$ has diameter equal to 2 . This implies that $B_{A}$ is not dentable, and that there is no any point of continuity for the identity mapping $\left(B_{A}\right.$, weak $) \rightarrow\left(B_{A}\right.$, norm $)$.
\end{abstract}

\section{Introduction}

Despite the importance of the Radon-Nikodym property in the theory of Banach spaces, many classical Banach spaces fail to enjoy that property. For instance, this is the case of infinite-dimensional $C(K)$-spaces and $C^{*}$ algebras. In this paper, we show that infinite-dimensional $C(K)$-spaces and $C^{*}$-algebras actually fail to fulfill most consequences of the Radon-Nikodym property, like the dentability of closed balls, or the existence of points of weak-norm continuity for the identity mapping on closed balls. In fact we prove that, if $A$ is an infinite-dimensional $C^{*}$-algebra, then every nonempty relatively weakly open subset of the closed unit ball of $A$ has diameter equal to 2 (Theorem 2.5). Our proof starts by revisiting the $C(K)$-space case, where the result is known [17], and passes through the consideration of some mathematical objects, called $J B^{*}$-triples (see $[\mathbf{1 5}]$ and $[\mathbf{1 6}]$ ), which become natural generalizations of $C^{*}$-algebras. In the result obtained for $J B^{*}$-triples, the infinite dimensionality must be replaced with the non hilbertizability (Proposition 2.4). We consider also the case of $J B$-algebras [13], where a similar result to that obtained for $J B^{*}$-triples is proven (Theorem $3.3)$.

1991 Mathematics Subject Classification. Primary 46B04, 46B22, 46L05, 46L70.

Partially supported by Junta de Andalucía grant FQM 0199. 


\section{The main result}

Throughout this paper $\mathbb{K}$ will mean the field of real or complex numbers. Let $X$ be a Banach space $X$ over $\mathbb{K}$. We denote by $S_{X}, B_{X}$, and $X^{*}$ the unit sphere, the closed unit ball, and the (topological) dual, respectively, of $X$. We denote by $n$ (respectively, $w$ ) the norm (respectively, weak) topology on $X$, and by $w^{*}$ the weak* topology of $X^{*}$. If $Y$ is another Banach space, $\mathcal{L}(X, Y)$ will stand for the space of all bounded linear operators from $X$ to $Y$.

Given a locally compact Hausdorff topological space $\Omega$, we denote by $C_{0}^{\mathbb{K}}(\Omega)$ the Banach space of all $\mathbb{K}$-valued continuous functions on $\Omega$ vanishing at infinity. When $\Omega$ is actually compact, we write as usual $C^{\mathbb{K}}(\Omega)$ instead of $C_{0}^{\mathbb{K}}(\Omega)$. The following lemma is a consequence of [17, Theorem 2]. For the sake of completeness, we give here a direct proof.

Lemma 2.1. Let $\Omega$ be a compact Hausdorff topological space, and let $X$ stand for $C^{\mathbb{K}}(\Omega)$. If there exists a nonempty relatively $w$-open subset of $B_{X}$ with diameter less than 2 , then $\Omega$ is finite.

Proof. Assume that $\Omega$ is infinite. Let $U$ be a nonempty relatively $w$ open subset of $B_{X}$. We are going to show that $\operatorname{diam}(U)=2$. Since $X$ is infinite-dimensional, there must exist some $x$ in $U \cap S_{X}$.

Consider first the case that the set of isolated points of $\Omega$ is infinite. Take a sequence $\left\{t_{n}\right\}_{n \in \mathbb{N}}$ of pair-wise different isolated points of $\Omega$, and, for $n$ in $\mathbb{N}$, define $x_{n} \in X$ by $x_{n}\left(t_{n}\right)=1, x_{n}\left(t_{n+1}\right)=-1$, and $x_{n}(t)=x(t)$ whenever $t$ belongs to $\Omega \backslash\left\{t_{n}, t_{n+1}\right\}$. Since $\left\{x_{n}\right\}_{n \in \mathbb{N}}$ lies in $B_{X}$ and converges pointwise to $x$, it follows from [10, Theorem VII.1] that $\left\{x_{n}\right\}_{n \in \mathbb{N}}$ actually converges weakly to $x$. Since $U$ is a relatively $w$-neighbourhood of $x$, we have that $x_{n}$ and $x_{n+1}$ belong to $U$ for $n$ big enough, and therefore $\operatorname{diam}(U)=2$.

Now consider the case that $x$ attains its norm at an accumulation point $t_{0}$ of $\Omega$. There is no loss of generality if we assume $x\left(t_{0}\right)=1$. Let $\varepsilon>0$. Then the set

$$
\omega_{0}:=\{t \in \Omega:|x(t)-1|<\varepsilon\}
$$

is open in $\Omega$ and contains $t_{0}$. Since $t_{0}$ is an accumulation point of $\Omega$, there is an infinite sequence $\left\{\omega_{n}\right\}_{n \in \mathbb{N}}$ of nonempty pair-wise disjoint open subsets of $\omega_{0}$. For $n \in \mathbb{N}$, take $t_{n} \in \omega_{n}$, and apply Uryson's lemma to pick $x_{n}$ in $X$ with $-\mathbf{1} \leq x_{n} \leq \mathbf{1}, x_{n}\left(t_{n}\right)=-1$, and $x_{n}(t)=1$ whenever $t \in \Omega \backslash \omega_{n}$. Since $\left\{x_{n}\right\}_{n \in \mathbb{N}}$ converges pointwise to $\mathbf{1},\left\{x x_{n}\right\}_{n \in \mathbb{N}}$ converges pointwise to $x$, and hence $\left\{x x_{n}\right\}_{n \in \mathbb{N}}$ actually converges weakly to $x$ because $\left\{x x_{n}\right\}_{n \in \mathbb{N}}$ lies in $B_{X}$. Let $m$ be in $\mathbb{N}$ such that $x x_{m}$ belongs to $U$. Then, since $t_{m}$ lies in $\omega_{0}$, we have

$$
\operatorname{diam}(U) \geq\left\|x-x x_{m}\right\| \geq\left|x\left(t_{m}\right)\left(1-x_{m}\left(t_{m}\right)\right)\right|=2\left|x\left(t_{m}\right)\right| \geq 2(1-\varepsilon) .
$$

By the arbitraryness of $\varepsilon$, we deduce $\operatorname{diam}(U)=2$.

To conclude the proof, consider the case that the set of isolated points of $\Omega$ is finite. Then we can write $X=(Y \times Z)_{\infty}$, where $Y=\left(\mathbb{K}^{p}\right)_{\infty}$ for 
some nonnegative integer number $p$, and $Z=C^{\mathbb{K}}(K)$ for some perfect compact Hausdorff topological space $K$. As a consequence, we have $\left(B_{X}, w\right)=$ $\left(B_{Y}, w\right) \times\left(B_{Z}, w\right)$. Since the coordinate projection $\pi_{Z}:\left(B_{X}, w\right) \rightarrow\left(B_{Z}, w\right)$ is open, it follows from the preceding paragraph that $\operatorname{diam}\left(\pi_{Z}(U)\right)=2$. Since $\pi_{Z}$ is contractive, we deduce $\operatorname{diam}(U)=2$.

LEMMA 2.2. Let $\Omega$ be a locally compact Hausdorff topological space, and let $X$ stand for $C_{0}^{\mathbb{K}}(\Omega)$. If there exists a nonempty relatively $w$-open subset of $B_{X}$ with diameter less than 2 , then $\Omega$ is finite.

Proof. Assume that there is a nonempty relatively $w$-open subset $U$ of $B_{X}$ with $\operatorname{diam}(U)<2$. Then $U$ contains a set $V$ of the form

$$
\left\{x \in B_{X}:\left|f_{i}\left(x-x_{0}\right)\right|<1 \forall i=1, \ldots, n\right\},
$$

for suitable $x_{0} \in B_{X}, n \in \mathbb{N}$, and $f_{1}, \ldots, f_{n} \in X^{*}$. Put

$$
V^{* *}:=\left\{z \in B_{X^{* *}}:\left|f_{i}\left(z-x_{0}\right)\right|<1 \forall i=1, \ldots, n\right\} .
$$

Since $V^{* *}$ is relatively $w^{*}$-open in $B_{X^{* *}}$, and $B_{X}$ is $w^{*}$-dense in $B_{X^{* *}}$, $V\left(=V^{* *} \cap B_{X}\right)$ is $w^{*}$-dense in $V^{* *}$. Therefore $V-V$ is $w^{*}$-dense in $V^{* *}-V^{* *}$, and consequently, by the lower semicontinuity of the norm of $X^{* *}$, we have $\operatorname{diam}\left(V^{* *}\right)=\operatorname{diam}(V) \leq \operatorname{diam}(U)<2$. Since $X^{* *}=C^{\mathbb{K}}(K)$ for a suitable compact Hausdorff topological space $K$, and $V^{* *}$ is a nonempty relatively $w$-open subset of $X^{* *}$, it follows from Lemma 2.1 that $K$ is finite. Now $X=X^{* *}$ is finite-dimensional, and hence $\Omega$ is finite.

The proof of Lemma 2.2 actually shows that, if $X$ is an infinite-dimensional real or complex Lindenstrauss space, then every nonempty relatively $w$-open subset of $B_{X}$ has diameter equal to 2 .

We recall that a $J B^{*}$-triple is a complex Banach space $J$ with a continuous triple product $\{\ldots\}: J \times J \times J \rightarrow J$ which is linear and symmetric in the outer variables, and conjugate-linear in the middle variable, and satisfies:

(1) For all $x$ in $J$, the mapping $y \rightarrow\{x x y\}$ from $J$ to $J$ is a hermitian operator on $J$ and has nonnegative spectrum.

(2) The main identity

$$
\{a b\{x y z\}\}=\{\{a b x\} y z\}-\{x\{b a y\} z\}+\{x y\{a b z\}\}
$$

holds for all $a, b, x, y, z$ in $J$.

(3) $\|\{x x x\}\|=\|x\|^{3}$ for every $x$ in $J$.

Concerning Condition 1 above, we also recall that a bounded linear operator $T$ on a complex Banach space $X$ is said to be hermitian if $\|\exp (\operatorname{ir} T)\|=1$ for every $r$ in $\mathbb{R}$.

$J B^{*}$-triples are of capital importance in the study of bounded symmetric domains in complex Banach spaces. Indeed, open balls in $J B^{*}$-triples are bounded symmetric domains, and every symmetric domain in a complex Banach space is biholomorphically equivalent to the the open unit ball of a 
suitable $J B^{*}$-triple (see $[\mathbf{1 5}]$ and $[\mathbf{1 6}]$ ). Examples of $J B^{*}$-triples are all $C^{*}$ algebras under the triple product

$$
\{x y z\}:=\frac{1}{2}\left(x y^{*} z+z y^{*} x\right),
$$

the spaces $\mathcal{L}\left(H_{1}, H_{2}\right)$ for arbitrary complex Hilbert spaces $H_{1}$ and $H_{2}$ (with triple product formally defined as in (2.1)), and the so-called spin factors. These are constructed from an arbitrary complex Hilbert space $(H,(\cdot \mid \cdot))$ of hilbertian dimension $\geq 3$, by taking a conjugate-linear involutive isometry $\sigma$ on $H$, and then by defining the triple product and the norm by

$$
\{x y z\}:=(x \mid y) z+(z \mid y) x-(x \mid \sigma(z)) \sigma(y)
$$

and

$$
\|x\|^{2}:=(x \mid x)+\sqrt{(x \mid x)^{2}-|(x \mid \sigma(x))|^{2}},
$$

respectively, for all $x, y, z$ in $H$.

Let $J$ be a $J B^{*}$-triple. A subtriple (respectively, triple ideal) of $J$ is a subspace $M$ of $J$ such that $\{M M M\} \subseteq M$ (respectively, $\{M J J\}+$ $\{J M J\} \subseteq M)$. We say that $J$ is simple if there are not triple ideals of $J$ other than $\{0\}$ and $J$. If $x$ is in $J$, and if the subtriple of $J$ generated by $x$ is finite-dimensional, then we say that $x$ is an algebraic element of $J$. The $J B^{*}$-triple $J$ is said to be algebraic if every element of $J$ is algebraic.

The proof of the next lemma involves minor changes on that of $[\mathbf{7}$, Theorem 1] or [8, Theorem D], and hence is omitted. Because of the conjugatelinear behaviour of triple products of $J B^{*}$-triples in the middle variable, and the essentially linear nature of the arguments in [7] and [8], the application of such arguments in our setting needs a formal consideration of "real algebraic" elements, being sure that "real algebraic" elements and (complex) algebraic elements coincide, thanks to the fact that $\mathbb{C}$ is finite-dimensional over $\mathbb{R}$.

Lemma 2.3. Let $J$ be a $J B^{*}$-triple. If there exists a nonempty open subset of $J$ consisting only of algebraic elements of $J$, then $J$ is algebraic.

Proposition 2.4. Let $J$ be a $J B^{*}$-triple such that there exists a nonempty relatively $w$-open subset of $B_{J}$ with diameter less than 2 . Then $J$ is a finite $\ell_{\infty}$-sum of closed simple triple ideals which are either finite-dimensional, spin factors, or of the form $\mathcal{L}\left(H_{1}, H_{2}\right)$ for suitable complex Hilbert spaces $H_{1}$ and $H_{2}$ with $\operatorname{dim}\left(H_{2}\right)<\infty$. Consequently, the Banach space of $J$ is hilbertizable.

Proof. Let $U$ be a nonempty relatively $w$-open subset of $B_{J}$ with $\operatorname{diam}(U)<2$. Let $x$ be in $J$. Denote by $J_{x}$ the closed subtriple of $J$ generated by $x$. It is well-known that there is a unique locally compact subset $S_{x}$ of ]0, $\infty$ [ and a surjective triple isomorphism $\phi_{x}: J_{x} \rightarrow C_{0}^{\mathbb{C}}\left(S_{x}\right)$ such that $S_{x} \cup\{0\}$ is compact and $\phi_{x}(x)$ is the inclusion mapping $S_{x} \rightarrow \mathbb{C}$ (see $[\mathbf{1 5}, 4.8],[\mathbf{1 6}, 1.15]$, and $[\mathbf{1 2}])$. Since surjective triple isomorphisms between 
$J B^{*}$-triples are isometries (see again $[\mathbf{1 6}]$ ), we can write $J_{x}=C_{0}^{\mathbb{C}}\left(S_{x}\right)$, in the sense of the isometric theory of Banach spaces. Now assume that $x$ actually lies in $U$. Then $U \cap J_{x}$ is a nonempty relatively $w$-open subset of $B_{J_{x}}$ with $\operatorname{diam}\left(U \cap J_{x}\right)<2$. It follows from Lemma 2.2 that $S_{x}$ is finite, and hence $x$ is an algebraic element of $J$. Since $x$ is arbitrary in $U$, and $U$ has nonempty $n$-interior in $J$, it follows from lemma 2.3 that $J$ is algebraic. Now, since $S_{x}$ is finite for every $x$ in $X$, the result follows easily from the results in [6] (see the concluding part of the proof of [6, Proposition 4.5] for details).

Since $C^{*}$-algebras are $J B^{*}$-triples, and they are finite-dimensional whenever their Banach spaces are reflexive [21], our main result follows straightforwardly from Proposition 2.4.

TheOREM 2.5. Let $A$ be a $C^{*}$-algebra such that there exists a nonempty relatively $w$-open subset of $B_{A}$ with diameter less than 2 . Then $A$ is finitedimensional.

Of course, the existence of a nonempty relatively $w$-open subsets of the closed unit ball with diameter less than 2, required in Theorem 2.5 (respectively, Proposition 2.4), is a condition much weaker than that of finite dimensionality (respectively, hilbertizability) arising in the conclusion of that result. Therefore we can obtain many other intermediate characterizations of the finite dimensionality (respectively, the hilbertizability) of $C^{*}$-algebras (respectively, $J B^{*}$-triples) in terms of the geometry of their closed unit balls. We do not state explicitly such characterizations, and only note as a hint that, for a Banach space $X$, each of the conditions 1 to 10 which follow implies the subsequent one:

(1) Finite-dimensionality

(2) Hilbertizability

(3) Superreflexivity

(4) Reflexivity

(5) The Radon-Nikodym property

(6) "Abundance" of denting points of $B_{X}$

(7) Existence of denting points of $B_{X}$

(8) Existence of slices of $B_{X}$ of arbitrarily small diameter

(9) Existence of nonempty relatively $w$-open subsets of $B_{X}$ of arbitrarily small diameter

(10) Existence of nonempty relatively $w$-open subsets of $B_{X}$ with diameter less than 2.

The implication $8 \Rightarrow 9$ above follows because, in fact, slices of $B_{X}$ are nonempty relatively $w$-open subsets of $B_{X}$. We note in addition that denting points of $B_{X}$ are points of $w-n$ continuity of the identity mapping on $B_{X}$, and that the mere existence of a point of $w-n$ continuity of the identity on $B_{X}$ implies condition 9 above. We recall that a slice of the closed unit ball 
of the Banach space $X$ is a set of the form

$$
S(X, f, \alpha):=\left\{x \in B_{X}: \Re e(f(x))>1-\alpha\right\}
$$

for some $f$ in $S_{X^{*}}$ and $\alpha>0$, that a denting point of $B_{X}$ is an element of $B_{X}$ such that there are slices of $B_{X}$ of arbitrarily small diameter containing it, and that condition 8 above is usually called dentability of $B_{X}$.

For some of the consequences of Theorem 2.5 suggested above, we are provided with a proof not involving the theory of $J B^{*}$-triples nor the nonassociative techniques in $[\mathbf{7}]$ or $[\mathbf{8}]$. This is the case of the following corollary.

COROLlary 2.6. Let $A$ be a $C^{*}$-algebra such that there exists a slice in $A$ with diameter less than 2 . Then $A$ is finite-dimensional.

Proof. Take a slice $S$ of $B_{A}$ with diameter less than 2. As in the proof of Lemma 2.2, we can produce a slice $S^{* *}$ of $B_{A^{* *}}$ with $\operatorname{diam}\left(S^{* *}\right)=\operatorname{diam}(S)$. Then, since $A^{* *}$ is a $C^{*}$-algebra with a unit, there is no loss of generality if we assume that $A$ has a unit $\mathbf{1}$. Let us denote by $U$ the set of all unitary elements of $A$. Since $B_{A} \backslash S$ is a convex, closed, and proper subset of $B_{A}$, and $\overline{c o}(U)=B_{A}[\mathbf{3}$, Theorem 30.2], there must exist $u$ in $U \cap S$. Then $S^{\prime}:=u^{*} S$ is a slice in $A$ containing $\mathbf{1}$ and whose diameter is less than 2. Let $h$ be a self-adjoint element of $A$, and let $A_{h}$ denote the closed subalgebra of $A$ generated by $\{h, \mathbf{1}\}$. Then we have $A_{h}=C^{\mathbb{C}}\left(\Omega_{h}\right)$, where $\Omega_{h}$ denotes the spectrum of $h$. Moreover, since $S^{\prime} \cap A_{h}$ is nonempty, it is in fact a slice in $A_{h}$ whose diameter is less than 2. According to Lemma 2.1, the spectrum of $h$ is finite. Since $h$ is an arbitrary self-adjoint element of $A$, Theorem 3.2.2 of [1] applies, giving that $A$ is finite-dimensional.

Let $X$ be a Banach space. For $u$ in $S_{X}$, we define the roughness of $X$ at $u, \eta(X, u)$, by the equality

$$
\eta(X, u):=\lim _{\sup }\|h\| \rightarrow 0 \frac{\|u+h\|+\|u-h\|-2}{\|h\|} .
$$

We remark that the absence of roughness of $X$ at $u$ (i.e., $\eta(X, u)=0$ ) is nothing but the Fréchet differentiability of the norm of $X$ at $u[\mathbf{9}$, Lemma I.1.3]. Given $\epsilon>0$, the Banach space $X$ is said to be $\epsilon$-rough if, for every $u$ in $S_{X}$, we have $\eta(X, u) \geq \epsilon$. We say that $X$ is rough whenever it is $\epsilon$-rough for some $\epsilon>0$, and extremely rough whenever it is 2-rough.

Corollary 2.7. Let $A$ be an infinite-dimensional von Neumann algebra. Then the predual of $A$ is extremely rough.

Proof. Let $A_{*}$ denote the predual of $A$, and let $u$ be in $S_{A_{*}}$. By Corollary 2.6, for every $\alpha>0$, the diameter of the slice $S(A, u, \alpha)$ is equal to 2. According to the proof of [9, Proposition I.1.11], this implies $\eta\left(A_{*}, u\right)=2$.

Corollary 2.7 is the unique predualitation we know of Theorem 2.5. A double predualization of that theorem (which in fact contains it) is really 
easy. Actually it is enough to invoke Theorem 2.5 after applying the argument in the proof of Lemma 2.2 .

Corollary 2.8. Let $X$ be an infinite-dimensional complex Banach space such that $X^{* *}$ is a $C^{*}$-algebra. Then every nonempty relatively $w$-open subset of $B_{X}$ has diameter equal to 2 .

\section{Other related results}

We begin this section by deriving again Theorem 2.5 from Proposition 2.4 , but now throughout a slightly longer way. This way is quite natural because the class of "ternary" generalizations of $C^{*}$-algebras (namely, that of $J B^{*}$-triples) contains the one of "binary" generalizations of $C^{*}$-algebras (namely, that of non-commutative $J B^{*}$-algebras).

Non-commutative Jordan algebras are defined as those algebras $A$ satisfying $(x y) x^{2}=x\left(y x^{2}\right)$ and $(x y) x=x(y x)$, for all $x, y$ in $A$. For an element $x$ in a non-commutative Jordan algebra $A$, let us denote by $U_{x}$ the mapping $y \rightarrow x(x y+y x)-x^{2} y$ from $A$ to $A$. By a non-commutative $J B^{*}$ algebra we mean a complete normed non-commutative Jordan complex algebra $A$ with a conjugate-linear algebra-involution $*$ satisfying $\left\|U_{x}\left(x^{*}\right)\right\|=\|x\|^{3}$ for every $x$ in $A$. As an example of the relevance of non-commutative $J B^{*}$-algebras in the general non-associative setting, let us mention that they are the unique (possibly non associative) complete normed complex algebras having an approximate unit bounded by one and whose open unit balls are bounded symmetric domains [14, Theorem 3.3], whereas $C^{*}$-algebras are the unique associative complete normed complex algebras satisfying the same two properties as above [14, Corollary 3.4].

We recall that a complex algebra $A$ is called quadratic if it has a unit $\mathbf{1}, A \neq \mathbb{C} \mathbf{1}$, and, for each $x$ in $A$, there are elements $t(x)$ and $n(x)$ of $\mathbb{C}$ such that $x^{2}-t(x) x+n(x) \mathbf{1}=0$. All quadratic non-commutative $J B^{*}$-algebras are hilbertizable [18, Theorem 3.2]. On the other hand, non-commutative $J B^{*}$-algebras whose Banach spaces are reflexive are perfectly determined [18, Theorem 3.5]. Keeping in mind such a determination, and the fact already commented that non-commutative $J B^{*}$-algebras are $J B^{*}$-triples in a natural way (see $[\mathbf{4}]$ and $[\mathbf{2 2}]$ ), the following result follows from Proposition 2.4 .

Proposition 3.1. Let $A$ be a non-commutative $J B^{*}$-algebra such that there exists a nonempty relatively w-open subset of $B_{A}$ with diameter less than 2. Then $A$ is a finite $\ell_{\infty}$-sum of closed simple ideals which are either finite-dimensional or quadratic. Consequently, the Banach space of $A$ is hilbertizable.

Alternative algebras are defined as those algebras $A$ satisfying $x^{2} y=x(x y)$ and $y x^{2}=(y x) x$ for all $x, y$ in $A$. By Artin's theorem $[\mathbf{2 4}$, Theorem 2.3.2], an algebra $A$ is alternative (if and) only if, for all $x, y$ in $A$, 
the subalgebra of $A$ generated by $\{x, y\}$ is associative. By an alternative $C^{*}$-algebra we mean a complete normed alternative complex algebra (say $A$ ) with a conjugate-linear algebra-involution $*$ satisfying $\left\|x^{*} x\right\|=\|x\|^{2}$ for all $x$ in $A$. Alternative $C^{*}$-algebras have also their own right to be considered as the non-associative counterparts of $C^{*}$-algebras. Indeed, GelfandNaimark axioms on a (possibly non associative) unital complex algebra $A$ imply that $A$ is alternative $[\mathbf{1 9}$, Theorem 14]. Since alternative algebras are non-commutative Jordan algebras, and, for elements $x, y$ in an alternative algebra, the equality $U_{x}(y)=x y x$ holds, it is not difficult to realize that alternative $C^{*}$-algebras become particular examples of non-commutative $J B^{*}$ algebras. Therefore, since simple quadratic alternative algebras are finitedimensional [24, Theorems 2.3.4 and 2.2.1], the next result follows from Proposition 3.1.

Proposition 3.2. Let $A$ be an alternative $C^{*}$-algebra such that there exists a nonempty relatively w-open subset of $B_{A}$ with diameter less than 2 . Then $A$ is finite-dimensional.

Since associative algebras are alternative, Proposition 3.2 contains Theorem 2.5 .

Our next and concluding goal in this section is to prove in the setting of $J B$-algebras similar results to that we have obtained for $J B^{*}$-triples, $C^{*}$ algebras, and non-commutative $J B^{*}$-algebras. $J B$-algebras are defined as those complete normed (commutative) Jordan real algebras $A$ satisfying $\|x\|^{2} \leq\left\|x^{2}+y^{2}\right\|$ for all $x, y$ in $A$. The basic reference for the theory of $J B$ algebras is the book of H. Hanche Olsen and E. Stormer [13]. By the main results in the papers of J. D. M. Wright $[\mathbf{2 3}]$ and M. A. Youngson $[\mathbf{2 2}], J B-$ algebras are in a bijective categorical correspondence with (commutative) $J B^{*}$-algebras. The correspondence is obtained by passing from each $J B^{*}$ algebra $A$ to its self-adjoint part $A_{s a}$.

Among the examples of $J B$-algebras, we cite the self-adjoint parts of $C^{*}$-algebras under the Jordan product $x \circ y:=\frac{1}{2}(x y+y x)$, and the so-called $J B$-algebra spin factors. These are the $J B$-algebras whose Banach spaces are of the form $(\mathbb{R} \oplus H)_{\ell_{1}}$, for an arbitrary real Hilbert space $H$ of dimension $\geq 2$, and whose products are defined by

$$
(\lambda+x)(\mu+y):=(\lambda \mu+(x \mid y))+(\lambda y+\mu x)
$$

(see $[\mathbf{1 3}$, Chapter 6]).

TheOREM 3.3. Let $A$ be a JB-algebra such that there exists a nonempty relatively w-open subset of $B_{A}$ with diameter less than 2 . Then $A$ is a finite $\ell_{\infty}$-sum of closed simple ideals which are either finite-dimensional, or JBalgebra spin factors. Consequently, the Banach space of $A$ is hilbertizable.

Proof. Let $U$ be a nonempty relatively $w$-open subset of $B_{A}$ with $\operatorname{diam}(U)<2$. Let $x$ be in $A$. Denote by $A_{x}$ the closed subalgebra of $A$ generated by $x$. Since Jordan algebras are power-associative $[\mathbf{1 3}$, Lemma 
2.4.5], if follows from [13, Theorem 3.2.2] that $A_{x}$ is isometrically algebraisomorphic to $C_{0}^{\mathbb{R}}\left(\Omega_{x}\right)$ for a suitable locally compact Hausdorff topological space $\Omega_{x}$. Assume for the moment that $x$ actually lies in $U$. Then $U \cap A_{x}$ is a nonempty relatively $w$-open subset of $B_{A_{x}}$ with $\operatorname{diam}\left(U \cap A_{x}\right)<2$, so that, by Lemma $2.2, \Omega_{x}$ is finite, and hence $A_{x}$ is finite-dimensional. Since $x$ is arbitrary in $U$, and $U$ has nonempty $n$-interior in $A$, it follows from either $\left[\mathbf{7}\right.$, Theorem 1] or $[\mathbf{8}$, Theorem $\mathrm{D}]$ that in fact $A_{x}$ is finite-dimensional for every $x$ in $A$. Now, for every $x$ in $A$, the set

$\sigma(x):=\left\{\lambda \in \mathbb{R}: x-\lambda \mathbf{1}\right.$ is not invertible in the unital hull of $\left.A_{x}\right\}$

is finite. Since, in particular, every nonzero point of $\sigma(x)$ is isolated for every $x \in A$, it follows from [5, Theorem $3.3(k \Rightarrow f)$ and Corollary 1.4] and [20, Theorem 5.4] that $A$ is a $c_{0}$-sum of topologically simple closed ideals which are either finite-dimensional, $J B$-algebra spin factors, or of the form $\mathcal{K}(H)_{s a}$ for some real, complex or quaternionic Hilbert space $H$. Since in fact $\sigma(x)$ is finite for every $x \in A$, the above $c_{0}$-sum must be finite, and the Hilbert spaces $H$ parameterizing the ideals $\mathcal{K}(H)_{s a}$, eventually arising in such a sum, must be finite-dimensional.

To conclude this paper, let us state the appropriate variants of Corollaries 2.7 and 2.8 in the setting of $J B^{*}$-triples and $J B$-algebras. We recall that the bidual of a $J B^{*}$-triple (respectively, $J B$-algebra) is a $J B^{*}$-triple (respectively, $J B$-algebra) in a natural way $[\mathbf{1 1}]$ (respectively, [13, Theorem 4.4.3]). Thus, $J B^{*}$-triples (respectively, $J B$-algebras) which are dual Banach spaces appear naturally. Such $J B^{*}$-triples (respectively, $J B$-algebras) are called $J B W^{*}$-triples (respectively, $J B W$-algebras). We note that the predual of a $J B W^{*}$-triple (respectively, $J B W$-algebra) is unique [2] (respectively, [13, Theorem 4.4.16]).

Corollary 3.4. Let $X$ be a $J B W^{*}$-triple or a $J B W$-algebra. Then the predual of $X$ is either hilbertizable or extremely rough.

Note that

Hilbertizability $\Rightarrow$ Superreflexivity $\Rightarrow$ Reflexivity $\Rightarrow$ Asplund

$\Rightarrow$ "Abundance" of points of Fréchet differentiability of the norm

$\Rightarrow$ Existence of points of Fréchet differentiability of the norm

$$
\Rightarrow \text { Non roughness, }
$$

so that the hilbertizability is scandalously incompatible with the extreme roughness.

Corollary 3.5. Let $X$ be a complex (respectively, real) Banach space such that $X^{* *}$ is a JB*-triple (respectively, JB-algebra). Then either $X$ is hilbertizable or all nonempty relatively $w$-open subsets of $B_{X}$ have diameter equal to 2 . 
Acknowledgements.- The authors are grateful to L. J. Bunce for fruitful comments about the matter of the paper.

\section{References}

[1] B. AUPETIT, Propriétés spectrales des algèbres de Banach. Lecture Notes in Math. 735, Springer-Verlag, Berlin, 1979.

[2] T. J. BARTON and R. M. TIMONEY, Weak*-continuity of Jordan triple products and applications. Math. Scand. 59 (1986), 177-191.

[3] F. F. BONSALL and J. DUNCAN, Numerical ranges II. London Math. Soc. Lecture Note Series 10, Cambridge University Press, Cambridge, 1973.

[4] R. B. BRAUN, W. KAUP, and H. UPMEIER, A holomorphic characterization of Jordan $C^{*}$-algebras. Math. Z. 161 (1978), 277-290.

[5] L. J. BUNCE, The theory and structure of dual JB-algebras. Math. Z. 180 (1982), 525-534.

[6] L. J. BUNCE and C. H. CHU, Compact operations, multipliers and RadonNikodym property in JB*-triples. Pacific J. Math. 153 (1992), 249-265.

[7] B. CUARTERO and J. E. GALÉ, Bounded degree of algebraic topological algebras. Commun. Algebra 22 (1994), 329-337.

[8] B. CUARTERO, J. E. GALÉ, A. RODRÍGUEZ, and A. M. SLINKO, Bounded degree of weakly algebraic topological Lie algebras. Manuscripta Math. 81 (1993), 129-139.

[9] R. DEVILLE, G. GODEFROY and V. ZIZLER, Smoothness and renormings in Banach spaces. Pitman Monographs and Surveys in Pure and Applied Math. 64, 1993.

[10] J. DIESTEL, Sequences and series in Banach spaces. Graduate Texts in Math. 92, Springer-Verlag, Berlin, 1984.

[11] S. DINEEN, The second dual of a $J B^{*}$-triple system. In Complex Analysis, Functional Analysis and Approximation Theory (ed. by J. Múgica), 67-69, NorthHolland Math. Stud. 125, North-Holland, Amsterdam-New York, 1986.

[12] Y. FRIEDMAN and B. RUSSO, Structure of the predual of a $J B W^{*}$-triple, $J$. Reine Angew. Math. 356 (1985), 67-89.

[13] H. HANCHE-OLSEN and E. STORMER, Jordan operator algebras. Monograph Stud. Math. 21, Pitman, 1984.

[14] A. KAIDI, A. MORALES, and A. RODRÍGUEZ, A holomorphic characterization of $C^{*}$ - and $J B^{*}$-algebras. Manuscripta Math. 104 (2001), 467-478.

[15] W. KAUP, Algebraic characterization of symmetric complex Banach manifolds. Math. Ann. 228 (1977), 39-64.

[16] W. KAUP, A Riemann mapping theorem for bounded symmetric domains in complex Banach spaces. Math. Z. 183 (1983), 503-529.

[17] O. NYGAARD and D. WERNER, Slices in the unit ball of a uniform algebra. Archiv Math. 76 (2001), 441-444.

[18] R. PAYÁ, J. PÉREZ, and A. RODRÍGUEZ, Type I factor representations of non-commutative $J B^{*}$-algebras. Proc. London Math. Soc. 48 (1984), 428-444.

[19] A. RODRÍGUEZ, A Vidav-Palmer theorem for Jordan $C^{*}$-algebras and related topics. J. London Math. Soc. 22 (1980), 318-332.

[20] A. RODRÍGUEZ, An approach to Jordan-Banach algebras from the theory of nonassociative complete normed algebras. In Algèbres de Jordan and algèbres normées non associatives, Ann. Sci. Univ. "Blaise Pascal", Clermont II, Sér. Math. 27 (1991), 1-57.

[21] S. SAKAI, Weakly compact operators on operator algebras. Pacific J. Math. 14 (1964), 659-664. 
[22] M. A. YOUNGSON, Non unital Banach Jordan algebras and $C^{*}$-triple systems. Proc. Edinburgh Math. Soc. 24 (1981), 19-31.

[23] J. D. M. WRIGHT, Jordan $C^{*}$-algebras. Michigan Math. J. 24 (1977), 291-302.

[24] K. A. ZHEVLAKOV, A. M. SLIN'KO, I. P. SHESTAKOV, and A. I. SHIRSHOV, Rings that are nearly associative. Academic Press, New York, 1982.

Universidad de Granada, Facultad de Ciencias. Departamento de Matemática APLICADA, 18071-GranADA (SPAIN)

E-mail address: juliobg@ugr.es

Universidad de Granada, Facultad de Ciencias. Departamento de Análisis Matemático, 18071-Granada (Spain)

E-mail address: glopezp@ugr.es

Universidad de Granada, Facultad de Ciencias. Departamento de Análisis Matemático, 18071-Granada (SPAin)

E-mail address: apalacio@ugr.es 